http://dx.doi.org/10.11646/zoosymposia.15.1.17

http://zoobank.org/urn:Isid:zoobank.org:pub:EADF80F0-4D93-4C38-9F30-DC4186E24EE7

\title{
Comparison of 2D SEM imaging with 3D micro-tomographic imaging for phylogenetic inference in brittle stars (Echinodermata: Ophiuroidea)
}

\author{
SABINE STÖHR $^{1 *}$, ELIZABETH G. CLARK ${ }^{2}$, BEN THUY $^{3} \&$ SIMON A. F. DARROCH $^{4}$ \\ ${ }^{1}$ Swedish Museum of Natural History, Dept of Zoology, Box 50007,10405 Stockholm, Sweden, sabine.stohr@nrm.se \\ ${ }^{2}$ Yale University, Dept of Geology and Geophysics, 210 Whitney Ave., New Haven, CT 06511, USA. Email: elizabeth.g.clark@yale.edu \\ ${ }^{3}$ Natural History Museum Luxembourg, Dept of Palaeontology, 25 rue Münster, 2160 Luxembourg City, Luxembourg. Email: \\ ben.thuy@mnhn.lu \\ ${ }^{4}$ Vanderbilt University, Dept of Earth and Environmental Science, 5726 Stevenson Center, Nashville, TN 37240, USA. Email: \\ simon.darroch@gmail.com \\ "Corresponding author
}

\begin{abstract}
Recent efforts to reconstruct the phylogeny of brittle stars (ophiuroids) have shown the need for more objective and reproducible data collection methods than the traditional visual examination and verbal description of morphological characters. Complex skeletal structures may be better understood in three dimensions than in two dimensions obtained from techniques like scanning electron microscopy (SEM). We test this hypothesis using three types of three-dimensional tomographic imaging methods-lab-based micro-CT, X-ray microscopy and synchrotron-based tomography - to examine the morphology of ophiuroid arms, and compare them with twodimensional data obtained from SEM. We describe the advantages and disadvantages of each instrument and set of parameters in terms of the ease and efficiency of data collection for morphometric analyses. We present new morphological observations obtained by digital sectioning of three-dimensional images that could not be achieved with SEM. Overall, our findings suggest that three-dimensional imaging has a high potential to address the gaps in knowledge of the internal ophiuroid skeleton, which will be pivotal to providing morphological characters that will aid in phylogenetic reconstructions.
\end{abstract}

Keywords. Morphology, taxonomy, micro-CT, synchrotron imaging, X-ray microscopy

\section{Introduction}

Reconstructing the phylogeny of ophiuroids has long been difficult due to limitations in available molecular techniques and insufficient understanding of their morphology (Stöhr 2012), until recent breakthroughs with both highly advanced molecular (O'Hara et al. 2014, 2017) and revised morphological (Thuy \& Stöhr 2016) methods. Historically, ophiuroids have been classified on the basis of externally observable morphological structures, such as outer scales and plates of disc and arms, the papillae of the jaws, granulation, spines or ornamentation of the disc scales, and arm spine shape and structure. Matsumoto $(1915,1917)$ also explored internal skeletal characters, such as the jaws, genital plates, and peristomial plates (among others) and was the first to use these to develop a classification of the Ophiuroidea. Murakami (1963) tested this classification using an examination of oral and dental plates but did not propose any major changes. Subsequently, Smith et al. (1995) performed a parsimony analysis of (mainly) morphological characters to produce the first modern phylogenetic hypothesis. However, this approach had limitations and proposed only minor changes to the prevailing classification (Stöhr 2012; Thuy \& Stöhr 2016). Over the last two decades, internal morphological studies (Martynov 2010; Thuy \& Stöhr 2011; Okanishi et al. 2017) and molecular analyses (O’Hara et al. 2017) have accumulated strong evidence suggesting that the previously accepted classification of ophiuroids does not 
reflect their true phylogenetic relationships. This has finally led to the proposal of a new phylogeny (O'Hara et al. 2017), and as a result a new higher classification (O'Hara et al. 2018), from class to family level.

The new classification scheme recognizes 33 families and six orders, a considerable increase on the 17 families and two orders recognized previously. However, this scheme still leaves many genera unplaced, due to a lack of both molecular and morphological data (O'Hara et al. 2018). Recently, Wilkie \& Brogger (2018) tested the phylogeny using an examination of peristomial plates, which have not been considered for their taxonomic information in over a century. They suggested that convergent evolution may have been driven by paedomorphosis and highlighted the need for more morphological data. Hendler (2018) proposed instead that allometric growth and adaptation to particular modes of feeding and locomotion may explain the evolution of the mouth skeleton. He also found previously unknown skeletal elements that may be useful for a phylogenetic analysis. A strictly molecular approach prevents the inclusion of extinct groups (which in all likelihood played an essential role in the evolution of this $\sim 480 \mathrm{Ma}$ old group), and their absence from the phylogeny may affect branch lengths and the branching pattern of the tree (Grantham 2004; Legg 2013; Ware \& Barden 2016). Since DNA cannot often be extracted from fossils, morphological characters are the only recourse for phylogenetic studies, and a large comparative dataset from recent forms is needed to place fossils in their phylogenetic context. Combining molecular and morphological data should ensure a higher accuracy in phylogenetic reconstructions; however, the morphological dataset recently produced by Thuy \& Stöhr $(2016,2018)$ is still too limited for such an approach (46 species, 130 characters). Nevertheless, morphological studies were instrumental in supporting the new classification (O'Hara et al. 2018).

It has been clear for over a century (e.g. Matsumoto 1917) that external morphology is insufficient to understand the evolution and phylogenetic relationships of brittle stars; however, the study of internal structures and micromorphology in this group has only recently been revived (Martynov 2010; Thuy \& Stöhr 2011; Okanishi et al. 2017; Hendler 2018). The few comprehensive studies focussing on the morphology of internal skeletal elements (ossicles) have relied on scanning electron microscopy (SEM) of individual ossicles, which are isolated by dissolving the entire animal in sodium hypochlorite (bleach), and which irreversibly damages the specimens (LeClair 1996; Martynov 2010; Thuy \& Stöhr 2011). This approach has nevertheless provided invaluable information on the shape and microstructure of skeletal elements, in particular the lateral arm plates which have been found to be phylogenetically highly informative (Martynov 2010; Thuy \& Stöhr 2011, 2016). Other skeletal structures that are expected to provide more information include the complex assemblage of elements that constitute the jaw apparatus and the genital plate/radial shield complex, but neither of these have been studied with modern methods.

Although undeniably useful, the images derived from SEM are two-dimensional, making it difficult to grasp the three-dimensional shape of the objects, their orientation, and how they articulate with associated parts. Attempts to overcome these limitations have been made by impregnation of samples in hard resin, followed by serial grinding to produce sectional photographs (Martynov et al. 2015). Although this approach has been valuable, it inevitably results in the destruction of the specimen, and thus prevents it being used with rare species or irreplaceable type material. Consequently, when a higher taxon is based on a rare type species that cannot be examined with these highly destructive techniques, this results in gaps in the character matrix. In part because of these limitations, Ziegler et al. (2018) emphasized the potential of three-dimensional imaging techniques for morphological studies as comparable and complimentary to molecular studies. Among non-destructive techniques, X-ray computed microtomography (micro-CT) has been used to study brooding species (Landschoff \& Griffiths 2015; MacKinnon et al. 2017) and arm mobility (Clark et al. 2018). For taxonomic research, micro-CT has so far only been applied to a single species of brittle star (Okanishi et al. 2017 ) but with promising results. Synchrotron-based imaging and other tomographic methods have successfully been used in sea urchins for a variety of applications (Ziegler 2012; Ziegler et al. 2016).

Traditional methods of morphological assessment are admittedly somewhat arbitrary and subjectivecharacters are identified by visual inspection of specimens or images and subsequently described in prose as precisely as possible. Mathematical methods such as geometric morphometrics may be more objective and reproducible, but they require standardized measuring techniques. Many of the characters found by Thuy \& Stöhr (2016) are too small for examination by light microscopy, which highlights the need for SEM and image analysis. However, with SEM, the objects are fixed in place on a holder and imaged. For comparable measurements, the objects need to be mounted in the exact same position and photographed from the same angles, which has proven difficult to achieve with strongly curved skeletal pieces. Three-dimensional models would overcome these problems. 
In this study we compared two-dimensional SEM and three-dimensional tomographic imaging techniques, and assessed their utility for large scale collection of morphological data for phylogenetic inference (specifically, the suitability of the resulting images for morphometric analysis). We focused on lateral arm plates because they have proven to be highly informative for phylogenetic reconstructions, are readily available in great numbers (both from living taxa and in microfossil samples), and have been studied extensively using SEM. We performed a case-study in which we used three types of X-ray microcomputed tomography to visualize the morphology of ophiuroid arm laterals:-1) a commercial lab-based micro-CT; 2) an X-ray microscope using X-ray beams coupled with an optical lens system; and, 3) a synchrotron beamline. These tests did not cover the full range of each instrument's capacity. Rather, our tests used standard parameters that may be refined with future experiments. This study therefore represents a preliminary investigation of the relative utility of these methods for phylogenetic purposes, and which may serve as a starting point for more exhaustive studies.

\section{Material and Methods}

We selected species from the three major clades found by Thuy \& Stöhr (2016). These are representatives of the three largest currently recognized orders (O'Hara et al. 2018), Ophiurida, Ophiacanthida, and Amphilepidida (Table 1). The samples were not saved after scanning but additional material is available from the collections at the Swedish Museum of Natural History. For reasons of comparability and availability, we chose arm pieces, which are among the best-studied parts of the ophiuroid skeleton. Initially, we imaged a larger sample of species through lab-based micro-CT, and then selected those with greatest morphological dissimilarity for this study and to cover phylogenetically widely-separated taxonomic groups. As the focus of this study was on hard skeletal parts, no staining techniques to visualize soft tissue were explored. Table 2 presents an overview of the tested tomographic parameter ranges. All tomographic images were compared to pre-existing SEM images by visual inspection in order to evaluate how well traditionally recognized features are represented. SEM was performed with a Hitachi S4300 and a FEI Quanta FEG 650, respectively, with a voltage of $5 \mathrm{kV}$ and a current of $10 \mu \mathrm{A}$.

TABLE 1. Data of the brittle star samples used for micro-CT, with register numbers for the museum lots from which these were taken and SEM stub register numbers. SMNH, Swedish Museum of Natural History.

\begin{tabular}{|c|c|c|c|}
\hline Order: Family & Species & Locality & Register no. \\
\hline $\begin{array}{l}\text { Ophiurida: } \\
\text { Ophiuridae }\end{array}$ & Ophiura sarsii & $\begin{array}{l}\text { Off Iceland, } 66^{\circ} 10.32^{\prime} \mathrm{N}, 12^{\circ} 01.29^{\prime} \mathrm{W}, 243 \mathrm{~m} \text {, } \\
\text { collector BIOICE sta. } 3247,14 / 07 / 2001\end{array}$ & $\begin{array}{l}\text { main sample not catalogued, } \\
\text { SEM sample SMNH-90652 }\end{array}$ \\
\hline $\begin{array}{l}\text { Ophiurida: } \\
\text { Ophiuridae }\end{array}$ & Ophiura sarsii & $\begin{array}{l}\text { Off Iceland, } 66^{\circ} 50.22^{\prime} \mathrm{N}, 16^{\circ} 16.26^{\prime} \mathrm{W}, 222-227 \\
\mathrm{~m} \text {, collector BIOICE sta. } 2673,15 / 07 / 1994\end{array}$ & SEM sample SMNH-45845 \\
\hline $\begin{array}{l}\text { Ophiurida: } \\
\text { Ophiuridae }\end{array}$ & $\begin{array}{l}\text { Ophiura } \\
\text { ophiura }\end{array}$ & $\begin{array}{l}\text { Off Iceland, } 63^{\circ} 27.95^{\prime} \mathrm{N}, 17^{\circ} 53^{\prime} \mathrm{W}, 126-130 \mathrm{~m} \text {, } \\
\text { collector BIOICE sta. } 2820,25 / 08 / 1995 \text {. }\end{array}$ & $\begin{array}{l}\text { SMNH-90278, SEM sample } \\
\text { SMNH-90259 }\end{array}$ \\
\hline $\begin{array}{l}\text { Ophiacanthida: } \\
\text { Ophiacanthidae }\end{array}$ & $\begin{array}{l}\text { Ophiacantha } \\
\text { bidentata }\end{array}$ & $\begin{array}{l}\text { Svalbard and Jan Mayen, Nordaustlandet, } 11 \\
\text { nautical miles SE of Isodden, } 79^{\circ} 28^{\prime} \mathrm{N}, 26^{\circ} 59^{\prime} \mathrm{E} \text {, } \\
200 \mathrm{~m} \text {, collector YMER } 80 \mathrm{sta} . \mathrm{MB} 04,07 / 07 / \\
1980\end{array}$ & $\begin{array}{l}\text { SMNH-169800, SEM sample } \\
\text { SMNH-90464 }\end{array}$ \\
\hline $\begin{array}{l}\text { Ophiacanthida: } \\
\text { Ophiotomidae }\end{array}$ & $\begin{array}{l}\text { Ophiotreta } \\
\text { valenciennesi }\end{array}$ & $\begin{array}{l}\text { New Caledonia, Lifou isl., off Cap des Pins, } \\
21^{\circ} 02.6^{\prime} \mathrm{S}, 167^{\circ} 31.6^{\prime} \mathrm{E}, 420-480 \mathrm{~m} \text {, collector S. } \\
\text { Stöhr, ep. Lifou } 2000 \text { sta CP1646, 24/11/2000 }\end{array}$ & $\begin{array}{l}\text { SMNH-109488, SEM sample } \\
\text { SMNH-121361 }\end{array}$ \\
\hline $\begin{array}{l}\text { Amphilepidida: } \\
\text { Amphiuridae }\end{array}$ & $\begin{array}{l}\text { Amphiura } \\
\text { chiajei }\end{array}$ & $\begin{array}{l}\text { Sweden, Gullmarsfjord, } 58^{\circ} 15.2^{\prime} \mathrm{N}, 11^{\circ} 26.8^{\prime} \mathrm{E} \text {, } \\
\text { collector O. Israelsson, } 02 / 05 / 2000\end{array}$ & $\begin{array}{l}\text { SMNH-90533, SEM sample } \\
\text { SMNH-90612 }\end{array}$ \\
\hline $\begin{array}{l}\text { Amphilepidida: } \\
\text { Ophiolepididae }\end{array}$ & $\begin{array}{l}\text { Ophiolepis } \\
\text { superba }\end{array}$ & Australia, Port Darwin, collector MacLeay, 1880 & $\begin{array}{l}\text { SMNH-170083, SEM sample } \\
\text { SMNH-174869 }\end{array}$ \\
\hline
\end{tabular}


TABLE 2. Micro-CT scanning parameters tested with brittle star samples.

\begin{tabular}{ll}
\hline Species and object & CT scanning parameters \\
\hline Ophiura ophiura arm & NSI Imagix: $28 \mathrm{kV}, 200 \mu \mathrm{A}, 4 \mathrm{~h} 24 \mathrm{~m}, 720$ images, voxel size $17.3 \mu \mathrm{m}$ \\
Ophiura sarsii arm & Zeiss Versa $520: 50 \mathrm{kV}$, le $1, \sim 2 \mathrm{~h}, 1004$ images, voxel size $3.5 \mu \mathrm{m}$ \\
Amphiura chiajei arm & Zeiss Versa $520: 50 \mathrm{kV}$, le $1, \sim 2 \mathrm{~h}, 100$ images, voxel size $2.5 \mu \mathrm{m}$ \\
Ophiolepis superba arm & NSI Imagix: $28 \mathrm{kV}, 200 \mu \mathrm{A}, 4 \mathrm{~h} 24 \mathrm{~m}, 720$ images, voxel size $22.4 \mu \mathrm{m}$ \\
Ophiotreta valenciennesi arm & NSI Imagix: $28 \mathrm{kV}, 200 \mu \mathrm{A}, 4 \mathrm{~h} 24 \mathrm{~m}, 720$ images, voxel size $17.3 \mu \mathrm{m}$ \\
Amphiura chiajei lateral arm plate & TOMCAT: $15 \mathrm{keV}, 10 \mathrm{x}$ mag, $17 \mu \mathrm{m}$ LSO:Tb scintillator screen, isotropic \\
& pixel $0.65 \mu \mathrm{m}, 1501$ projections, 1275 images, 7m21s \\
\hline
\end{tabular}

Lab-based micro-CT: Dried arm pieces were placed in Eppendorf plastic vials, and locked in place with a thin plastic sheet. Three groups of between five and six vials were scanned together to optimize time, using the North Star Imaging Imagix X-ray computed microtomography (micro-CT) scanner, housed at Vanderbilt University (Tennessee, USA). Three species were selected from the resulting datasets (Table 1), and 2D digital slices were extracted from the reconstructions (see below) using myVGL 3.1 (Macintosh version).

$X$-ray microscopy: Dried arm pieces of Ophiura sarsii Lütken, 1858 and Amphiura chiajei Forbes, 1843 were placed in Eppendorf plastic vials and scanned separately in an X-ray microscope (XRM) Zeiss Versa 520 , housed at the 4D imaging lab in Lund, Sweden.

Synchrotron tomography: size limitations of the chosen instrument did not allow the use of intact arm pieces. Therefore, lateral arm plates of Ophiacantha bidentata (Bruzelius, 1805) and A. chiajei were isolated by immersing an arm piece in bleach until all soft tissue had been dissolved, washing the skeletal elements with tap water and letting them air dry. A single plate from each species was then glued onto its own brass stub with nail polish, and synchrotron radiation X-ray tomographic microscopy (SRXTM) was performed at the TOMCAT beamline at the Swiss Light Source, Paul Scherrer Institute, Switzerland.

Volume rendering and $3 D$ visualization: The digital $2 \mathrm{D}$ slices produced by each instrument were combined into tiff-stacks. To reduce the file size, the XRM and SRXTM image stacks were cropped by removing empty space, whereas in the case of the micro-CT scans each specimen was cropped from the image stacks of the group scans. The SRXTM files were also binned in all directions (x, y, z) by a factor 2 to further reduce the stack size, which increased the isotropic voxel size from 0.65 to 1.3 micron. All of these preparations were performed with ImageJ/Fiji (Abramoff et al. 2004). The specimens were then reconstructed and visualized through volume rendering with the open source software package Drishti (Limaye 2012). The specimens from the micro-CT scans were also visualized as surface rendered 3D digital representations, using the software VGStudio MAX, and each arm piece and two lateral arm plates were isolated digitally with segmentation functions of the software. The XRM visualizations were digitally sliced in Drishti to reveal features of the inner skeleton, such as nerve and water canals, and muscle fibres. For illustrative purposes, 2D representations were saved as images from the $3 \mathrm{D}$ visualizations.

All seven tomographic datasets (tiff stacks) and 10 SEM images (in total about 2 GB) have been deposited in a Morphobank project (\#3386) available at http://morphobank.org/permalink/?P3386.

\section{Results}

With all three tomographic techniques, the 3D visualizations obtained with both surface rendering and volume rendering techniques provide an understanding of the overall shape of skeletal parts and how they interconnect with each other (Figs 1-3). For instance, in Ophiolepis superba H.L. Clark, 1915 (Fig. 1), the large size of the outer arm plates relative to the small vertebra and the precise fit of these parts is clearly visible in the $3 \mathrm{D}$ view (Fig. 1C, E). The angled shape of the thick laterals is obvious in the 3D view (Fig. 1C, E) but difficult to appreciate in SEM images (Fig. 1A, B, F). 


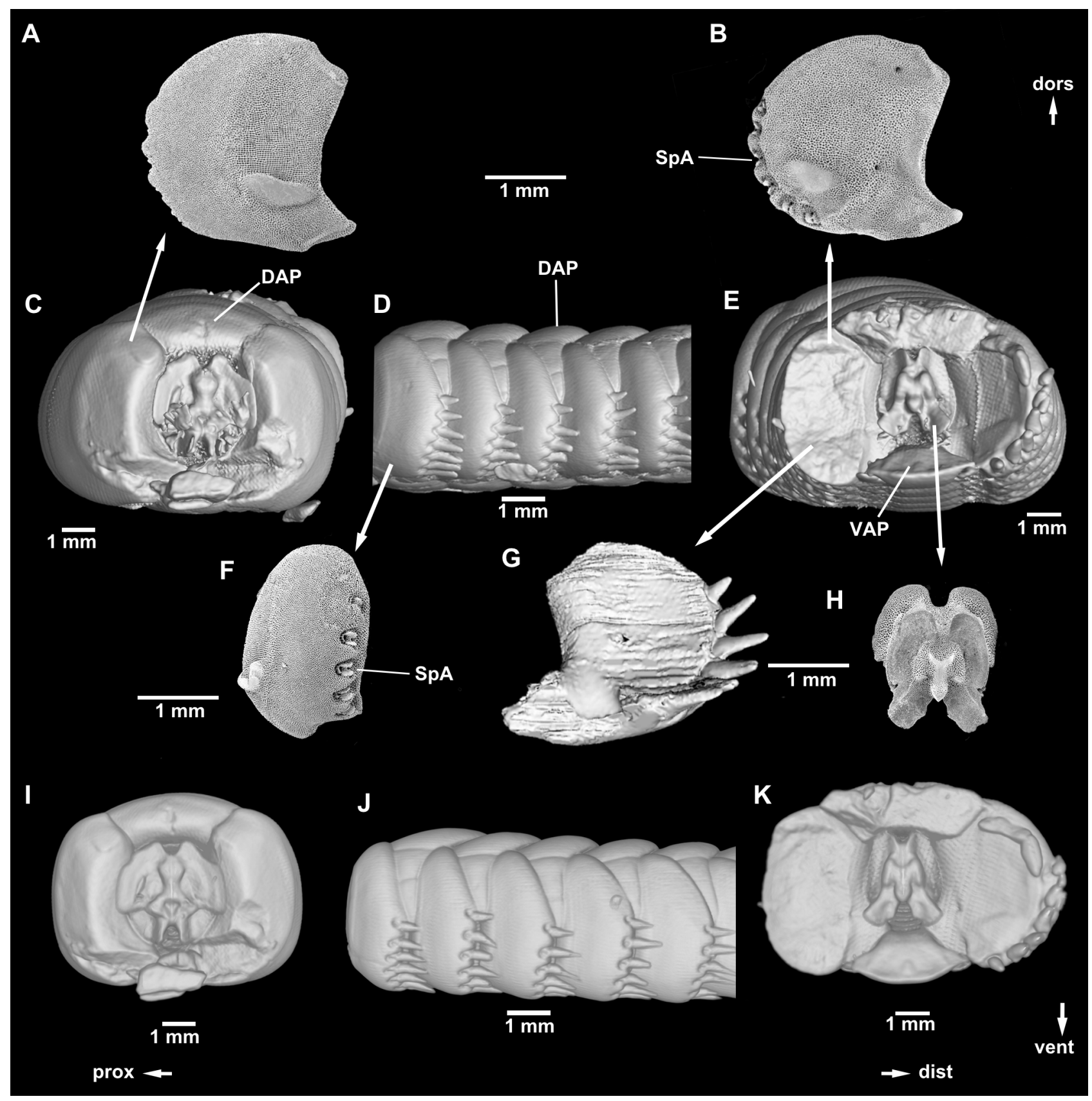

FIGURE 1. Comparison of results from several imaging techniques as described in the text, arm pieces of Ophiolepis superba, A-B, SEM, lateral arm plate, external (A) and internal (B) aspect, C-E, 3D visualizations from micro-CT, surface rendered, C, joint, proximal aspect, D, arm in lateral view, E, joint, distal aspect, F, lateral plate, proximal view, SEM, G, lateral plate, internal view, micro-CT, F, vertebra, distal aspect, I-K, same as C-E, volume rendered. DAP, dorsal arm plate, SpA, spine articulation, VAP, ventral arm plate. Surface renderings were created by digitally generating watertight polygonal meshs over the surface of the structures of interest in VG Studio MAX.

In the micro-CT images we obtained, the surface of the arms is smoothed out by the surface rendering technique, such that the stereom structure is obscured, and micro-structures such as arm spine articulations are not as well defined as in SEM images (Ophiotreta valenciennnesi (Lyman, 1878), Fig. 2D-H). The volume rendered images (Figs. 1I-K, 2A-C) do not show stereom details either and the shape of plates and smaller features are not as well defined as in the surface rendered images. This may be explained by the low resolution (large voxel sizes) chosen for these experiments, which is a good option for visualization of overall shape but not for fine details such as articulations and stereom structure. However, imaging with different parameters than we used may result in increased resolution. 


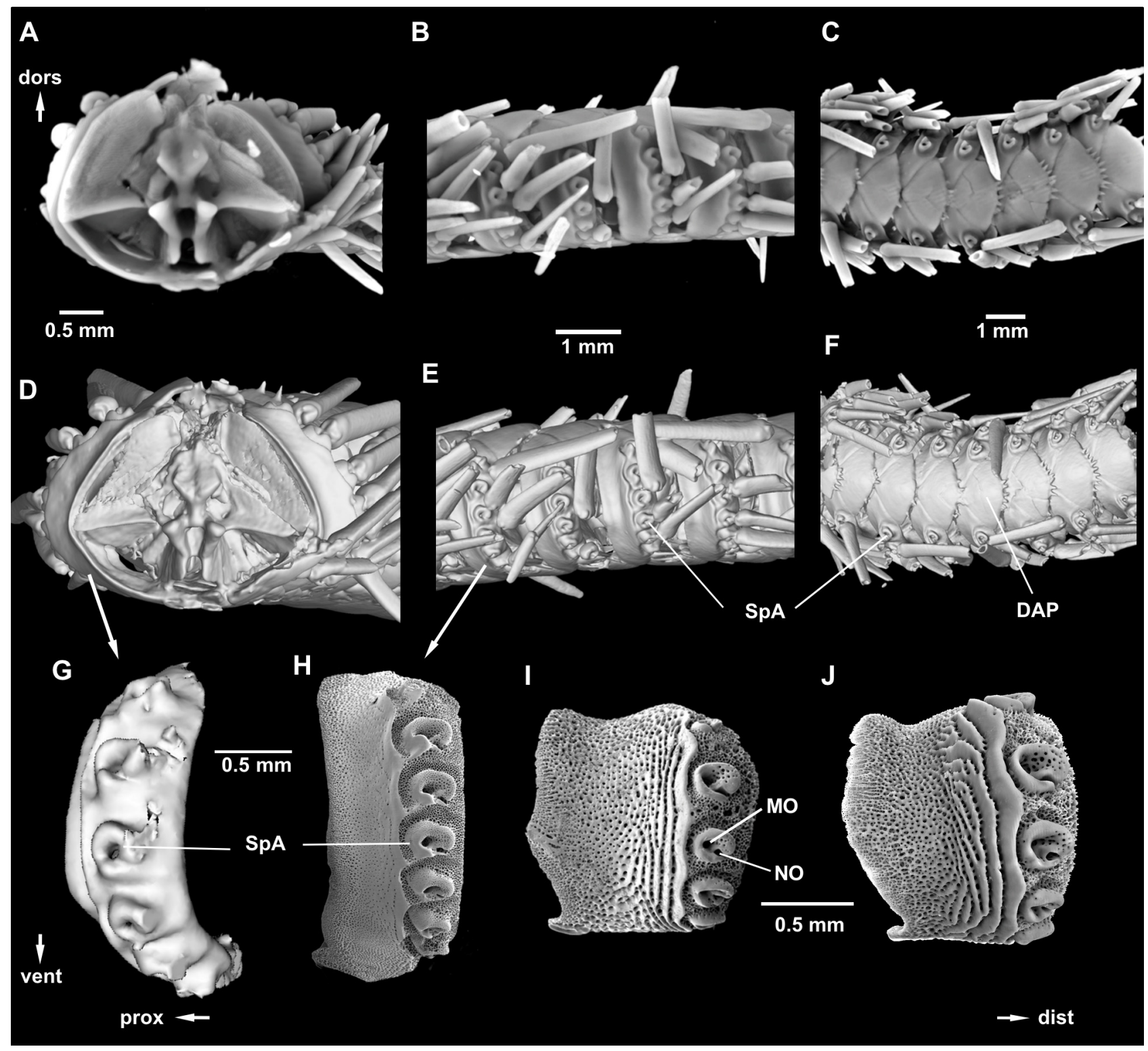

FIGURE 2. 3D images by micro-CT, volume rendered (A-C), surface rendered (D-G), and SEM image (E) of Ophiotreta valenciennesi. A, arm joint, proximal view, B, arm, lateral view, C, arm, dorsal view, D-F, same as A-C, G, digitally segmented lateral arm plate from $3 \mathrm{D}$ visualization, $\mathrm{H}$, lateral arm plate in external view. I, SEM image of lateral arm plate of Ophiacantha bidentata. J, 3D image of a lateral arm plate of $O$. bidentata, by synchrotron imaging. DAP, dorsal arm plate, VAP, ventral arm plate, $\mathrm{SpA}$, spine articulation.

With XRM and the smaller voxel sizes chosen, our images (Ophiura sarsii, Fig. 3A) captured fine details (stereom structure, spine articulations), while providing 3D information that is similar to the lab-based microCT (Ophiura ophiura (Linnaeus, 1758), Fig. 3D, E) but superior to SEM images (Fig. 3F). The XRM also captured dried intervertebral muscle fibres in Amphiura chiajei (Fig. 4C, D), without special preparation. We also observed lateral water canal branches inside the vertebrae (Fig. 5) and nerve canals in the arm spine articulations (Fig. 5B, C, D, F, G) in the digitally sectioned volumes. The spine articulation lobes in A. chiajei seem to be smooth and compact externally as observed in the SEM image (Fig. 4B) but then appear hollow when digitally sectioned (Fig. 5D, E). The digitally sectioned vertebrae of $A$. chiajei present a dark central longitudinal line as evidence of an interlocking rather than fused connection between the left and right halves (Fig. 5H). 


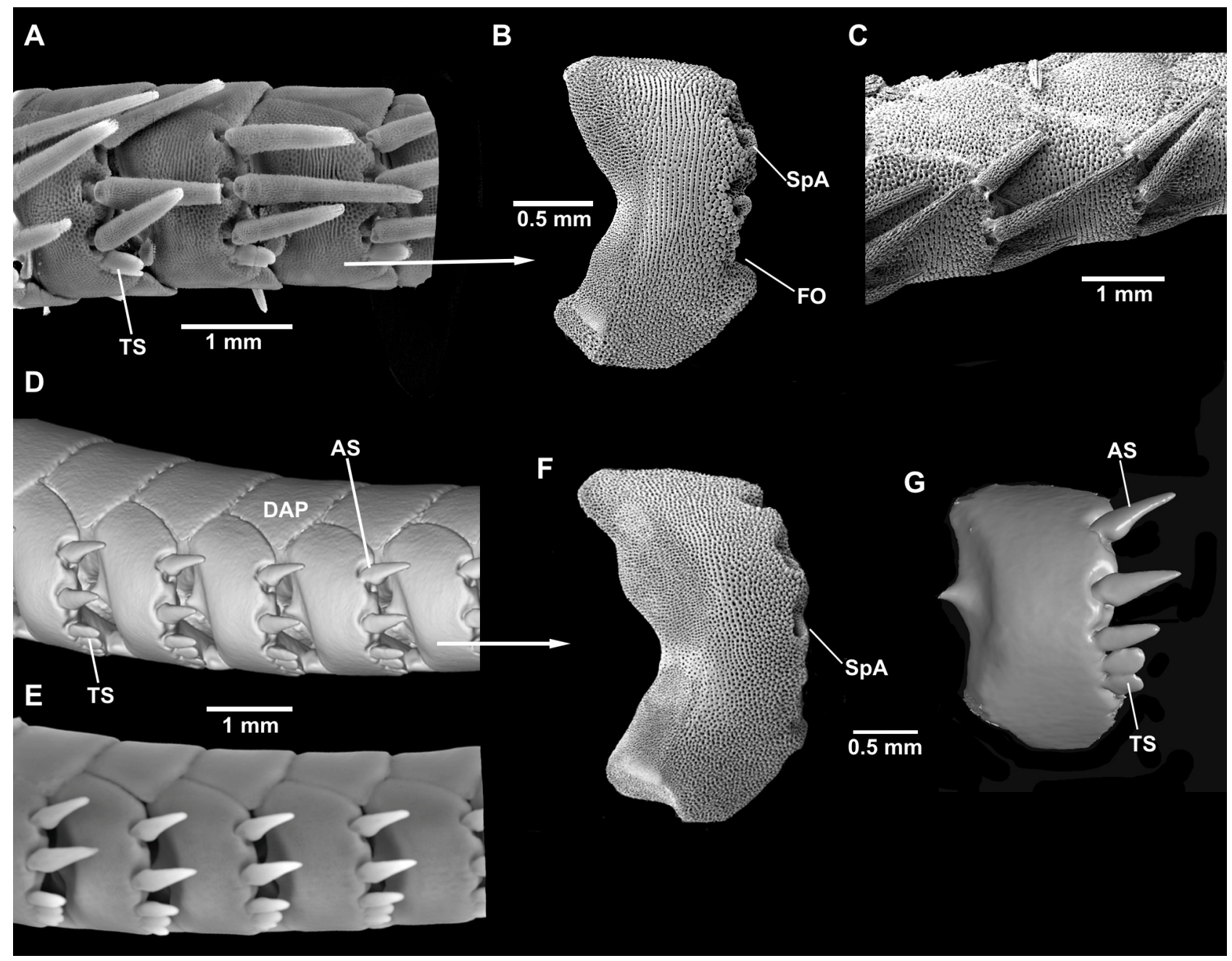

FIGURE 3. Comparison of images of Ophiura sarsii $(\mathrm{A}-\mathrm{C})$ and Ophiura ophiura $(\mathrm{D}-\mathrm{G})$. A, volume rendered 3D visualization, X-ray microscopy, arm in lateral view, B, lateral arm plate, external view, SEM, C, lateral arm, SEM, D, surface rendered $3 \mathrm{D}$ visualization, micro-CT, lateral arm, E, the same as D, volume rendered, F, lateral arm plate, SEM, G, digitally segmented arm plate from D. DAP, dorsal arm plate, SpA, spine articulation.

Our SRXTM images have the highest resolution and show excellent details of shape and stereom structure (Ophiacantha bidentata, Fig. 2J, Amphiura chiajei, Fig. 4A), barely distinguishable from SEM images in the 2D representations (Figs 2I, 4A).

\section{Discussion}

\section{Morphological observations}

Okanishi et al. (2017) found that intervertebral muscles in Asteronyx loveni Müller \& Troschel, 1842 can be observed by micro-CT without special preparation, and our observations confirm this. In both studies, dried material was used and Okanishi et al. (2017) suggested that dry muscles may have shrunk and compacted, making them more easily to detect with x-ray (although we note that experiments with wet preserved material are needed to verify this). Okanishi et al. (2017) showed for the first time how the lateral branches of the radial water canal (inside the ambulacral groove) penetrate the vertebrae on each side to connect to the tube feet. By virtually sectioning our volume rendered visualizations of the XRM scans, we have revealed these for two more species (O. sarsii and A. chiajei). Water canals are part of the basal bauplan of Ophiuroidea, and there seems to be little variation in the position or shape of these structures. Nerve canals in the arm spine articulations were also observed by Okanishi et al. (2017) and examined here by virtually sectioning the XRM 
scan of $A$. chiajei and $O$. sarsii. It appears that the nerve canal ends blindly in the lateral arm plate in these species, as we did not find corresponding openings on the inside of the plates and the openings vanish when followed through sequential slices. In addition, we observed the suture line connecting the left and right halves of each vertebra and found a small gap that suggests that the stereom interlocks but does not fuse. This is consistent with the observation of unfused vertebrae in paedomorphic and juvenile forms (Stöhr \& Martynov 2016). Based on SEM images that show a smooth, unperforated surface of the spine articulation lobes in A. chiajei, we assumed that they were composed of compact stereom. The virtually sectioned images suggest instead that these structures are hollow. This illustrates that three-dimensional imaging has a high potential for the discovery of previously unknown features.
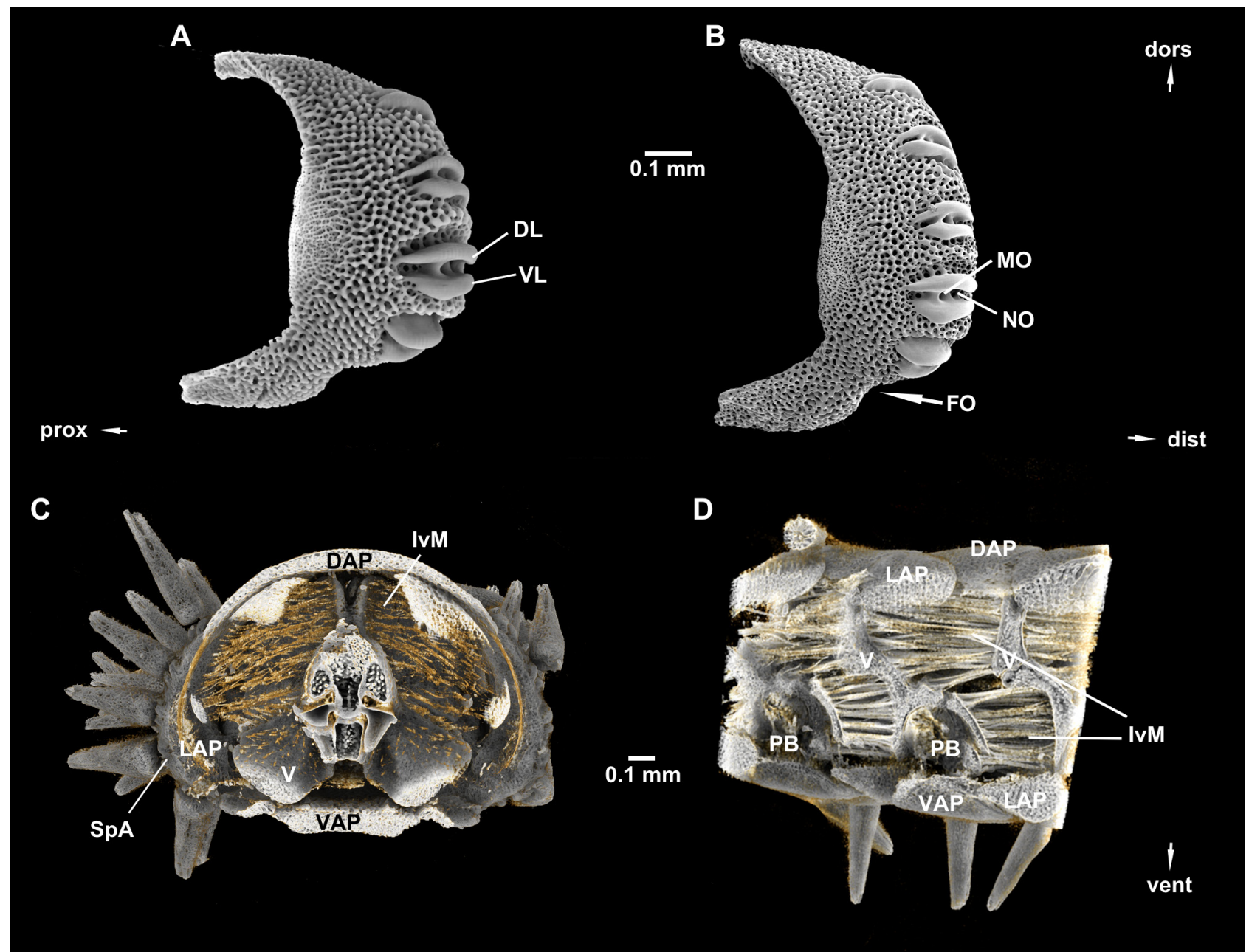

FIGURE 4. Amphiura chiajei, A, 3D image of lateral arm plate, external aspect, synchrotron imaging, B, same by SEM, C, XRM image, arm joint, distal view, note the intervertebral muscle fibres, D, same in lateral view. DAP, dorsal arm plate, DL, dorsal lobe, FO, foot opening, IvM, intervertebral muscle fibres, LAP, lateral arm plate, MO, muscle opening, NO, nerve opening, PB, podial basin, SpA, spine articulation, V, vertebra, VAP, ventral arm plate, VL, ventral lobe.

\section{Evaluation of images}

Our tests suggest that the advantage of the 3D imaging techniques over SEM is that a single scan of an arm piece can provide morphological information in three dimensions. For example, the complete articulated skeleton can be viewed from any angle by digital rotation, and single plates can be digitally isolated by segmentation. However, digital segmentation is time consuming, and on the digitally isolated lateral arm plates we noticed image artefacts that may be mistaken for micro-structures (Fig. 1G, 2G, 3G), which are absent in the corresponding SEM images (Figs. 1B, 2H, 3F). All three CT instruments require minimal or no preparation, although the chosen SRXTM beamline required destructive sampling of arm laterals due to its sample size limits. Choosing a different beamline that can manage larger samples may remedy that issue. By comparison, SEM is a highly destructive technique, because the ophiuroid skeleton is covered by integument 
that needs to be removed (usually by bleaching) for SEM but not for X-ray tomography. In addition, covering all aspects of an object with SEM requires several preparations with different orientations, and a series of images.

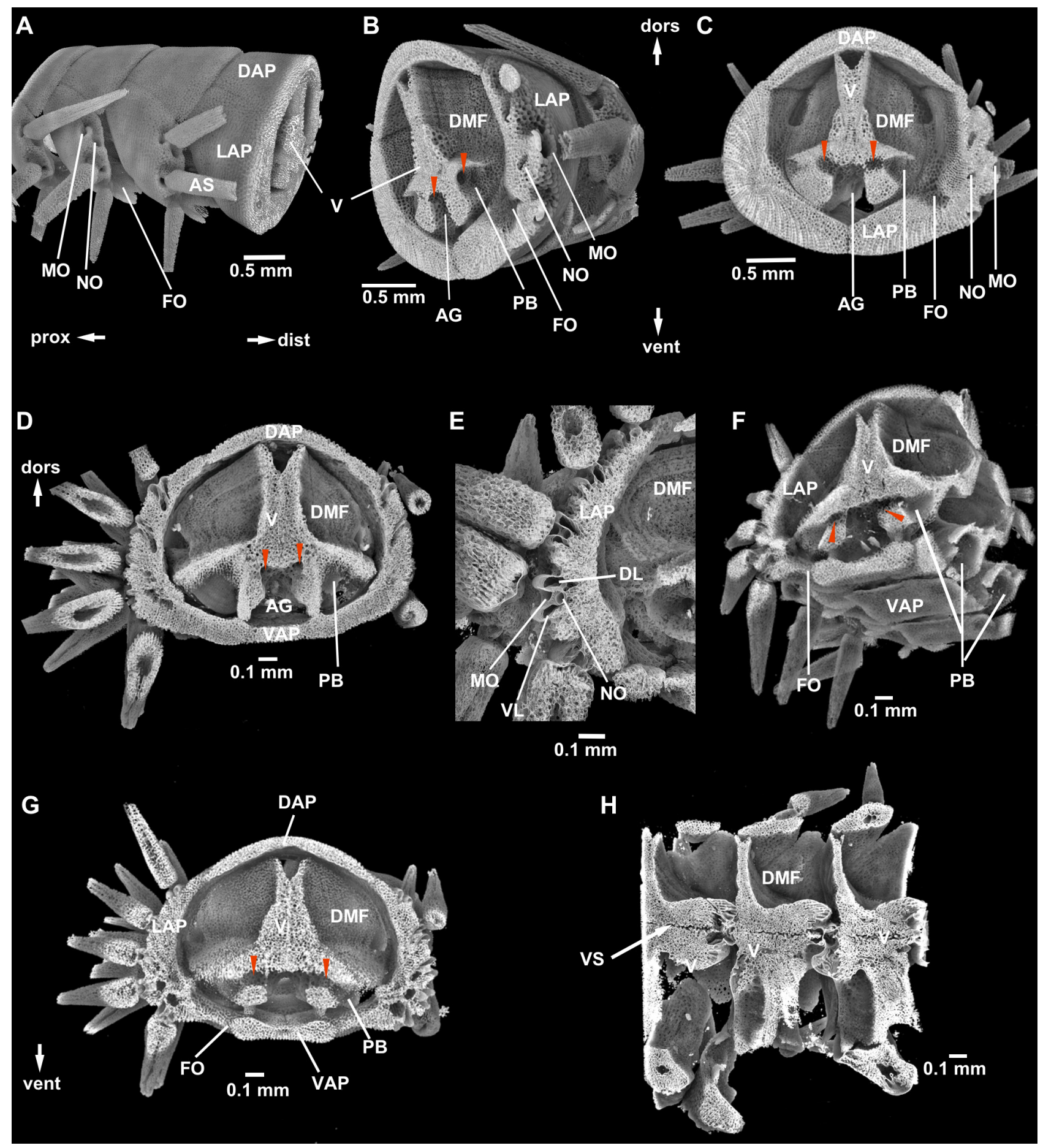

FIGURE 5. Digitally sectioned arms from XRM scans, volume rendered, of Ophiura sarsii (A-C) and Amphiura chiajei (DH). Red arrowheads point to openings for lateral water canals. A, lateral aspect, B, laterodistal aspect sliced to expose vertebra with closed lateral water canals, $\mathrm{C}$, distal aspect, sliced to expose lateral canals, D, distal aspect, sliced past lateral water canal openings to expose arm spine articulation openings, E, magnification of distal section, spine openings exposed, F, ventrodistal view, distally sliced to open up lateral water canals, with left arm surface sliced off, G, distal aspect with water canals opened, $\mathrm{H}$, ventral view, partially sliced off. For details of lateral arm plates compare fig. 4A, B. AG, ambulacral groove (radial water canal opening), AS, arm spine, DAP, dorsal arm plate, DL, dorsal lobe, DMF, distal muscle flange of vertebra, FO, foot opening through ventral and lateral plates, LAP, lateral arm plate, MO, muscle opening, NO, nerve opening, PB, podial basin, V, vertebra, VAP, ventral arm plate, VL, ventral lobe, VS, vertebral suture. 
For phylogenetic inference, in addition to descriptive trait analysis, data should also be collected from images using morphometric analyses; we evaluate different types of imaging techniques with respect to their potential for collecting data valuable for morphometric analyses here. The potential for geometric morphometric methods to be used in brittle star phylogenetic inference has not been explored, but promising attempts have been made in sea urchins (David \& Laurin 1996), and so it seems likely that they will have similarly successful application in brittle stars. Lab-based micro-CT volume renderings had relatively lower resolution and may only be suitable for morphometric analyses on larger structures, such as whole lateral plates or spines. Micro-structures such as spine articulations are not well enough defined to attempt shape comparisons, although changing the scanning parameters to achieve higher resolution might improve this. In addition, the speed of image acquisition (i.e., scanning) was quite slow (approximately 4 hours for the ophiuroid ossicles in this case), which we attempted to compensate for by scanning groups of samples. This approach also requires extensive digital processing of the resulting images ('segmenting') to separate individual arms, which initially overlap in the visualizations. In contrast, our XRM and synchrotron images provide fine detail in shape and structure, and seem equally suitable for morphometric analysis of microstructures, such as spine articulations and stereom structure. Furthermore, synchrotron imaging was by far the fastest method (6-8 minutes per sample) in our tests, although this is possibly due to the small size of the scanned object (lateral arm plate). However, the facilities are not as readily available, may require traveling between countries, and may be limited with regard to object size and preservation medium. Lastly, XRM instruments are readily available in a laboratory environment, provide sufficient resolution, and are flexible with regard to object size (up to $30 \mathrm{~cm}$ according to manufacturer) and preservation medium. Scan speeds were higher than with micro-CT, and samples can be kept in any medium (fluid or dry) as long as there is sufficient contrast between medium and object. XRM is thus a genuinely non-destructive technique, and may even provide information on soft tissue (muscles) without the need for staining (although again we note that further experiments are needed to confirm this). We did not test scanning of fossils with any of these instruments, and it is possible that results will differ due to the (frequently) greater density of fossilized material.

Processing the images of the digitized specimens is often necessary to isolate specific skeletal plates. The plates of interest must be designated automatically or manually in the $3 \mathrm{D}$ visualization software in the $3 \mathrm{D}$ volume render or the 2D image slices through a "segmentation" process. Visualization of lateral ossicles scanned articulating with other arm plates may not be ideal subjects for automatic segmentation, as small differences in density between adjacent skeletal plates or mounting material prevents the software programs from accurately identifying the target objects. The process can be time-consuming, although the higher resolution of the XRM did provide better visibility of structures (and thus easier segmentation) than microCT. An alternative would be to scan plates that have already been isolated; these may be difficult to examine with a lab-based micro-CT due to their small size but would be possible with XRM or synchrotron imaging. However, in order to understand the position of the parts in relation to each other, whole-body scans are needed (which can be performed with either of the three tomographic instrument types depending on the specifications of the machine). For rare and irreplaceable material, a whole-body scan, followed by digital segmentation may be the only realistic option.

This study was limited to lateral arm plates as these have been heavily studied by previous workers, and are thus ideally suited for a comparison of methods. They may, however, not be ideal subjects for automated segmentation, as they are surrounded by overlapping plates of similar shape and density. In addition, arm plates are heavily affected by growth changes not only between specimens, but also along the entire length of the arm (brittle star arms grow new joints at the tip, and these differ in shape from older joints in basal position-see Thuy \& Stöhr 2011). When analysing arm plates, therefore, the exact position along an arm needs to be considered, in addition to the ontogenetic stage and size of the animal. Other structures potentially hold equally valuable information that has not been unlocked and may be less prone to these problems. Particularly promising (but difficult to study) are the genital plates, which articulate with the radial shields from below. Genital plates have until now been examined solely with SEM (Martynov 2010) or light microscopy (Matsumoto 1917). These plates are non-symmetrical with complex morphology of the articulations, making it difficult to position them on the SEM stub in exactly the same orientation every time for comparable results between species. It is also difficult to recognize the shapes of knobs, pits, grooves etc. using two-dimensional images. Finally, the dissection of genital plates requires the destruction of the disc. Tomographic imaging techniques and $3 \mathrm{D}$ visualization represents a solution to these problems. 


\section{Conclusions}

For taxonomic revisions and species descriptions, morphological characters are still widely used, alone or in combination with molecular data. Inferring a phylogeny from morphological data alone is challenging, and although promising results have been achieved (Thuy \& Stöhr 2016, 2018), limitations of the approach have become apparent. The two-dimensional images derived from SEM are insufficient to fully understand the shape of skeletal elements, and thus are failing to unlock the full spectrum of the morphological complexity of the brittle star skeleton. Three-dimensional visualizations of these structures have not, thus far, been widely used. In this study we perform a preliminary exploration of 3D micro-computed tomographic imaging techniques in order to assess advantages and disadvantages compared to $2 \mathrm{D}$ imaging.

The results suggest that all of the tested parameters do, however, have the potential to provide new insights, and depending on the specific research question being addressed (e.g. finding morphological characters and tracing them through time, or collecting data for phylogenetic inference), a suitable method may be chosen. However, on the basis of our investigation, XRM seems ideally suited to address the gaps in knowledge of the internal skeletal (and possibly associated muscles and nerves) morphology of brittle stars. In our study, XRM served as a non-destructive, high resolution, and high contrast 3D microscopic imaging technique with wide applicability. Moreover, this method allows for imaging of a wide range of specimen sizes, possesses a relatively fast rate of image acquisition, and is an instrument type that is increasingly available in laboratory environments. In the future, we suggest that optimal parameters for fast throughput of large numbers of samples need to be developed. Establishing these parameters will lay the groundwork for a computational pipeline for image processing, which, in combination with automated methods for geometric morphometric analysis (see, for example, Hsiang et al. 2018) may eventually be able to handle the large amounts of data needed for phylogenetic inference.

\section{Acknowledgments}

We wish to express our gratitude to the organizers of the 16th International Echinoderm Conference, Tatsuo Oji, Tatsuo Motokawa, Mieko Komatsu, Toshihiko Fujita, Yukio Agatsuma, and Ken'ichi Kanazawa, for a wonderful meeting and for editing this proceedings volume. We are very grateful to Stephen Hall (4D imaging Lab, Lund), for providing test scans of brittle star arms with a Zeiss Xradia 520. We thank the Paul Scherrer Institute, Villigen, Switzerland for provision of synchrotron radiation beamtime at the TOMCAT beamline X02DA of the Swiss Light Source (projects 20171476). We are grateful to Federica Marone and Else Marie Friis for help at the beamline and SRXTM analyses. Many thanks to Alexander Ziegler for very valuable suggestions and information that greatly improved the manuscript. S. Stöhr thanks Gudmundur Gudmundsson, Icelandic Institute of Natural History, for the loan of BIOICE material, and Philippe Bouchet for the opportunity to collect brittle stars at Lifou (financed by the Totalfina Elf foundation). The SEM examination of lateral arm plates was funded by Synthesys project SE-TAF-2674 to B. Thuy. Thanks are also due to Robert Asher for the excellent video tutorials on the use of Drishti, which he published on Youtube (available at https://www.youtube.com/channel/UCIpfqI07qSnCSBxe3JvdgSg).

\section{References}

Abramoff, M.D., Magalhaes, P.J. \& Ram, S.J. (2004) Image processing with ImageJ. Biophotonics International, $11,36-42$.

Clark, E.G., Hutchinson, J.R., Darroch, S.A.F., Mongiardo Koch, N., Brady, T.R., Smith, S.A. \& Briggs, D.E.G. (2018) Integrating morphology and in vivo skeletal mobility with digital models to infer function in brittle star arms. Journal of Anatomy, 233, 696-714. https://doi.org/10.1111/joa.12887

David, B. \& Laurin, B. (1996) Morphometrics and cladistics: measuring phylogeny in the sea urchin Echinocardium. Evolution, 50, 348-359. https://doi.org/10.2307/2410806

Grantham, T. (2004) The role of fossils in phylogeny reconstruction: Why is it so difficult to integrate paleobiological and neontological evolutionary biology? Biology \& Philosophy, 19, 687-720.

https://doi.org/10.1007/s10539-005-0370-z 
Hendler, G. (2018) Armed to the teeth: a new paradigm for the buccal skeleton of brittle stars (Echinodermata: Ophiuroidea). Contributions in Science, 526, 189-311.

Hsiang, A.Y., Nelson, K., Elder, L.E., Sibert, E.C., Kahanamoku, S.S., Burke, J.E., Kelly, A., Liu, Y. \& Hull, P.M. (2018) AutoMorph: Accelerating morphometrics with automated 2D and 3D image processing and shape extraction. Methods in Ecology and Evolution, 9, 605-612. https://doi.org/10.1111/2041-210X.12915

Landschoff, J. \& Griffiths, C. (2015) Three-dimensional visualisation of brooding behaviour in two distantly related brittle stars from South African waters. African Journal of Marine Science, 37, 533-541. https://doi.org/10.2989/1814232X.2015.1095801

LeClair, E.E. (1996) Arm joint articulations in the ophiuran brittlestars (Echinodermata: Ophiuroidea): a morphometric analysis of ontogenetic, serial, and interspecific variation. Journal of Zoology, London, 240, 245-275. https://doi.org/10.1111/j.1469-7998.1996.tb05283.x

Legg, D. (2013) The impact of fossils on arthropod phylogeny. $\mathrm{PhD}$ thesis. Imperial College.

Limaye, A. (2012) Drishti: a volume exploration and presentation tool. Proceedings of SPIE 8506. Developments in X-Ray Tomography VIII, 85060X. https://doi.org/10.1117/12.935640

MacKinnon, R.B., Landschoff, J. \& Griffiths, C.L. (2017) Seasonality and 3D-visualization of brooding in the hermaphroditic ophiuroid Amphiura capensis. Invertebrate Biology, 136, 146-158. https://doi.org/10.1111/ivb.12164

Martynov, A., Ishida, Y., Irimura, S., Tajiri, R., O’Hara, T. \& Fujita, T. (2015) When Ontogeny Matters: A New Japanese Species of Brittle Star Illustrates the Importance of Considering both Adult and Juvenile Characters in Taxonomic Practice. PLOS ONE, 10, e0139463. https://doi.org/10.1371/journal.pone.0139463

Martynov, A.V. (2010) Reassessment of the classification of the Ophiuroidea (Echinodermata), based on morphological characters. I. General character evaluation and delineation of the families Ophiomyxidae and Ophiacanthidae. Zootaxa, 2697, 1-154.

https://doi.org/10.11646/zootaxa.2697.1.1

Matsumoto, H. (1915) A new classification of the Ophiuroidea: with descriptions of new genera and species. Proceedings of the Academy of Natural Sciences, Philadelphia, 67, 43-92.

Matsumoto, H. (1917) A monograph of Japanese Ophiuroidea, arranged according to a new classification. Journal of the College of Science, Imperial University, Tokyo, 38, 1-408.

Murakami, S. (1963) The dental and oral plates of Ophiuroidea. Transactions of the Royal Society of New Zealand, Zoology, 4, $1-48$.

O’Hara, T.D., Hugall, A.F., Thuy, B. \& Moussalli, A. (2014) Phylogenomic Resolution of the Class Ophiuroidea Unlocks a Global Microfossil Record. Current Biology, 24, 1874-1879.

https://doi.org/10.1016/j.cub.2014.06.060

O’Hara, T.D., Hugall, A.F., Thuy, B., Stöhr, S. \& Martynov, A.V. (2017) Restructuring higher taxonomy using broad-scale phylogenomics: The living Ophiuroidea. Molecular Phylogenetics and Evolution, 107, 415-430. https://doi.org/10.1016/j.ympev.2016.12.006

O’Hara, T.D., Stöhr, S., Hugall, A.F., Thuy, B. \& Martynov, A. (2018) Morphological diagnoses of higher taxa in Ophiuroidea (Echinodermata) in support of a new classification. European Journal of Taxonomy, 1-35. https://doi.org/10.5852/ejt.2018.416

Okanishi, M., Fujita, T., Maekawa, Y. \& Sasaki, T. (2017) Non-destructive morphological observations of the fleshy brittle star, Asteronyx loveni using micro-computed tomography (Echinodermata, Ophiuroidea, Euryalida). ZooKeys, 663, 1-19. https://doi.org/10.3897/zookeys.663.11413

Smith, A.B., Paterson, G.L.J. \& Lafay, B. (1995) Ophiuroid phylogeny and higher taxonomy: morphological, molecular and palaeontological perspectives. Zoological Journal of the Linnean Society, 114, 213-243. https://doi.org/10.1006/zjls.1995.0024

Stöhr, S. (2012) Ophiuroid (Echinodermata) systematics-where do we come from, where do we stand and where should we go? Echinoderm Research 2010: Proceedings of the Seventh European Conference on Echinoderms, Göttingen, Germany, 2-9 October 2010. Zoosymposia, 7, 147-161.

Stöhr, S. \& Martynov, A. (2016) Paedomorphosis as an Evolutionary Driving Force: Insights from Deep-Sea Brittle Stars. PLOS ONE, 11, e0164562. https://doi.org/10.1371/journal.pone.0164562

Thuy, B. \& Stöhr, S. (2011) Lateral arm plate morphology in extant brittle stars (Echinodermata) and its application in micropalaeontology. Zootaxa, 3013, 1-47. 
https://doi.org/10.11646/zootaxa.3013.1.1

Thuy, B. \& Stöhr, S. (2016) A New Morphological Phylogeny of the Ophiuroidea (Echinodermata) Accords with Molecular Evidence and Renders Microfossils Accessible for Cladistics. PLoS ONE, 11, e0156140.

https://doi.org/10.1371/journal.pone.0156140

Thuy, B. \& Stöhr, S. (2018) Unravelling the origin of the basket stars and their allies (Echinodermata, Ophiuroidea, Euryalida). Scientific Reports, 8, 8493 .

https://doi.org/10.1038/s41598-018-26877-5

Ware, J.L. \& Barden, P. (2016) Incorporating fossils into hypotheses of insect phylogeny. Current Opinion in Insect Science, $18,69-76$.

https://doi.org/10.1016/j.cois.2016.10.003

Wilkie, I.C. \& Brogger, M.I. (2018) The peristomial plates of ophiuroids (Echinodermata: Ophiuroidea) highlight an incongruence between morphology and proposed phylogenies. PLOS ONE, 13, e0202046. https://doi.org/10.1371/journal.pone.0202046

Ziegler, A. (2012) Broad application of non-invasive imaging techniques to echinoids and other echinoderm taxa. Zoosymposia, 7, 53-70.

Ziegler, A., Bock, C., Ketten, D.R., Mair, R.W., Mueller, S., Nagelmann, N., Pracht, E.D. \& Schröder, L. (2018) Digital ThreeDimensional Imaging Techniques Provide New Analytical Pathways for Malacological Research. American Malacological Bulletin, 36, 248-274. https://doi.org/10.4003/006.036.0205

Ziegler, A., Lenihan, J., Zachos, L.G., Faber, C. \& Mooi, R. (2016) Comparative morphology and phylogenetic significance of Gregory's diverticulum in sand dollars (Echinoidea: Clypeasteroida). Organisms Diversity \& Evolution, 16, $141-166$. https://doi.org/10.1007/s13127-015-0231-9 